\title{
KLF5 promotes apoptosis induced by phorbol ester as an effector of the autocrine factor TNF $\alpha$ in LNCaP prostate cancer cells
}

\author{
QI SHI ${ }^{1}$, JING JIA ${ }^{1}$, KE HUI $^{1}$, YANG GAO ${ }^{1}$, SHAN XU ${ }^{1,2}$, BING GUAN $^{1}$, XIAOSHUANG TANG ${ }^{1}$, \\ XINYANG WANG ${ }^{1,2}$, DALIN HE ${ }^{1,2}$ and PENG GUO ${ }^{1,2}$ \\ ${ }^{1}$ Department of Urology, The First Affiliated Hospital of Xi'an Jiaotong University; ${ }^{2}$ Oncology Research Lab, \\ Key Laboratory of Environment and Genes Related to Diseases, Ministry of Education, \\ Xi'an Jiaotong University, Xi'an, Shaanxi 710061, P.R. China
}

Received September 17, 2015; Accepted April 4, 2017

DOI: $10.3892 / 01.2017 .6293$

\begin{abstract}
Krüppel-like factor 5 (KLF5) is frequently deleted and inactivated in prostate cancer, and exerts tumor-suppressing function in prostate cancer cells. However, the function of KLF5 in the apoptosis of prostate cancer cells remains unclear. In the present study, the effect of KLF5 on phorbol 12-myristate 13-acetate (PMA)-induced apoptosis was investigated in prostate cancer LNCaP cells. It was demonstrated that PMA induced the expression of KLF5 at the mRNA and protein level. To identify whether KLF5 regulates the activity of the downstream pathway, stable KLF5 knockdown or overexpression cell lines were constructed with lentivirus harboring shRNA targeting KLF5 or full-length KLF5 in LNCaP cells. Knockdown of KLF5 significantly decreased PMA-induced apoptosis, while cell apoptosis was significantly increased following KLF5 overexpression compared with the corresponding control groups. Consistently, expression of cleaved poly(ADP-ribose) polymerase and caspase- 3 induced by PMA was decreased following KLF5 knockdown and increased following KLF5 overexpression. Using the control medium from cells treated with PMA, it was demonstrated that KLF5 is required for the control medium to induce apoptosis. c-Jun N-terminal kinase (JNK) activity is essential for the apoptosis induced by PMA. It was revealed that knockdown of KLF5 decreased, while overexpression of KLF5 increased the phosphorylation of JNK induced by PMA and control medium treatment. Furthermore, inhibition of tumor necrosis factor $\alpha$ (TNF $\alpha$ ) decreased KLF5 expression and significantly decreased cell apoptosis induced by PMA, and control medium. This data indicates that KLF5 is
\end{abstract}

Correspondence to: Professor Peng Guo or Professor Dalin He, Department of Urology, The First Affiliated Hospital of Xi'an Jiaotong University, 277 West Yan-Ta Road, Xi'an, Shaanxi 710061, P.R. China

E-mail: guopeng661@mail.xjtu.edu.cn

E-mail: dalinhexjtu@163.com

Key words: KLF5, PMA, TNFa, JNK, prostate cancer essential for the apoptosis induced by PMA in LNCaP prostate cancer cells. Furthermore, KLF5 is essential for activity of the autocrine factor TNF $\alpha$, which is secreted by cells treated with PMA and mediates the function of PMA-induced apoptosis through regulating the activity of JNK signaling pathway. These results provide novel insights into the complexity of the signaling pathways regulating apoptosis in prostate cancer cells, which could aid in the development of novel treatments for patients with prostate cancer.

\section{Introduction}

Prostate cancer is the most prevalent type of male cancer in Western countries and represents the fourth most common type of cancer worldwide (1). Prostate cancer growth is initially androgen-dependent; hence the gold standard of treatment is hormone-ablation therapy with anti-androgens and/or androgen-deprivation therapies (2). However, the efficacy of these treatments in the majority of patients is short-lived and the cancer typically recurs in a more aggressive form, termed castrate-resistant prostate cancer, which remains challenging to treat, and is fatal.

Phorbol esters, which are activators of protein kinase $\mathrm{C}$ isozymes, can trigger distinct cellular processes, including skin carcinogenesis in mice and migration of human glioma cells (3). Despite their well-known tumor-promoting effects, phorbol esters can also induce apoptosis in several types of cancer cells, including prostate cancer cells (4). Studies have demonstrated that phorbol 12-myristate 13-acetate (PMA), one of the most active phorbol esters, can induce apoptosis in LNCaP prostate cancer cells (5). The prostate cancer LNCaP cell line is considered to be a suitable model to investigate the mechanism of apoptosis induced by phorbol esters in prostate cancer cells $(4,6,7)$, in which the release of death factors, including tumor necrosis factor $\alpha(\mathrm{TNF} \alpha)$, are necessary $(7,8)$.

The Krüppel-like transcription factor 5 (KLF5/IKLF/ BTEB2), a type of zinc-finger transcription factor, has important roles in various cellular processes, including in cell proliferation, cell cycle progression and apoptosis (9-11). Several previous studies have demonstrated that KLF5 acts as an oncogene in different types of cancer, including in bladder and breast cancer $(10,12)$. Patients with increased 
KLF5 expression in breast cancer exhibit shorter disease-free survival times, and increased KLF5 expression aids in cancer cell proliferation and tumorigenesis $(10,12)$. However, KLF5 has also been demonstrated to act as a tumor suppressor under certain conditions, including JNK activation-induced apoptosis in esophageal squamous cell cancer (13). The loss of KLF5 expression has frequently been reported in prostate cancer; furthermore, several studies have demonstrated that the deletion and inactivation of KLF5 promotes tumor growth in prostate cancer $(14,15)$. However, the precise function of KLF5 in prostate cancer remains unclear.

$\mathrm{TNF} \alpha$ is a type of inflammatory cytokine that regulates normal cell and tissue functions, including immune responses, hematopoiesis and morphogenesis; however, it has also been implicated in deleterious processes, such as tumorigenesis, transplant rejection, septic shock, viral replication and bone resorption (16). The expression of $\mathrm{TNF} \alpha$ leads to the activation of mitogen-activated protein kinase (MAPK) cascades, including the ERK1/2, p38, c-Jun N-terminal kinase (JNK) signaling pathways (16). TNFa has been reported to induce apoptosis in the LNCaP cell line derived from prostate cancer cells $(17,18)$, and is an essential molecule involved in the autocrine loop through PMA-induced apoptosis in LNCaP cells $(7,8)$. Previous studies have demonstrated that TNF $\alpha$ can upregulate the KLF5 mRNA and protein levels (19), which results in cell apoptosis via activation of the JNK signaling pathway in esophageal cancer (13). Therefore, it has been suggested that KLF5 participates in the PMA-induced TNFa autocrine loop, which may be important in apoptosis of LNCaP cells.

In the present study, the role of PMA in the activation of KLF5 in LNCaP cells was investigated. Furthermore, the role of KLF5 in the regulation of the JNK signaling pathway was determined.

\section{Materials and methods}

Cell culture and reagents. Human prostate cancer $\mathrm{LNCaP}$ cells and human embryonic kidney 293T cells were obtained from the American Type Culture Collection (Manassas, VA, USA). The LNCaP cells were cultured in RPMI-1640 (Thermo Fisher Scientific, Inc., Waltham, MA, USA) supplemented with $10 \%$ fetal bovine serum (FBS; Hyclone; GE Healthcare Life Sciences, Logan, UT, USA) and 293T cells were cultured in Dulbecco's Modified Eagle's Medium (DMEM; Thermo Fisher Scientific, Inc.) supplemented with $10 \% \mathrm{FBS}$ at $37^{\circ} \mathrm{C}$ with $5 \% \mathrm{CO}_{2}$ in a humidified incubator. TNF $\alpha$ and anti-human TNF- $\alpha$ antibody were purchased from PeproTech, Inc. (Rocky Hill, NJ, USA). PMA was purchased from Sigma-Aldrich (Merck KGaA, Darmstadt, Germany). These reagents were dissolved in $0.1 \%$ bovine serum albumin (Sigma-Aldrich; Merck $\mathrm{KGaA}$ ) and stored at $-20^{\circ} \mathrm{C}$. The antibody directed against human KLF5 has been previously described (15).

Lentivirus preparation. PLKO.1 lentiviral vectors were used to package encoding short hairpin RNAs (shRNAs) with the sequence, 5'-GGTTACCTTACAGTATCAACA-3'. To generate the lentivirus, PAX2, VSV-G and the aforementioned plasmids were co-transfected into $90 \%$ confluent $293 \mathrm{~T}$ cells using Lipofectamine 2000 reagent (Invitrogen; Thermo Fisher
Scientific, Inc., Waltham, MA, USA), according to the manufacturer's protocol. Lentivirus expressing KLF5 was produced using full-length KLF5 from the pcDNA3-KLF5 plasmid (20), which was sub-cloned into LV5 vectors according to the manufacturer's protocol (Shanghai GenePharma Co., Ltd., Shanghai, China).

Western blot analysis. Western blot analysis was performed using a previously described method (11). A total of $2 \times 10^{6} \mathrm{LNCaP}$ cells were washed once with cold PBS and lysed in radioimmunoprecipitation assay buffer $(50 \mathrm{mM}$ Tris $\mathrm{pH} 8.0$, $150 \mathrm{mM} \mathrm{NaCl}, 0.1 \%$ SDS, $1 \% \mathrm{NP}-40$, and $0.5 \%$ sodium deoxycholate) containing $1 \%$ protease inhibitor cocktail and $1 \mathrm{mM}$ phenylmethanesulfonyl fluoride (Sigma-Aldrich; Merck KGaA). The concentration of protein was detected using a Bradford Assay Protein Quantification kit (Abcam, Cambridge, UK). A total of $30 \mu \mathrm{g}$ protein was separated by $12 \%$ SDS-PAGE and transferred onto nitrocellulose membranes. The membranes were blocked with $5 \%$ skimmed milk reconstituted in Tris-buffered saline with $0.1 \%$ Tween-20 (pH 7.6) at room temperature for $1 \mathrm{~h}$, and washed with PBS three times, followed by incubation at $4^{\circ} \mathrm{C}$ overnight with primary antibody. The primary antibodies used were as follows: GAPDH (1:10,000; cat. no. KC-5G4; Shanghai KangChen Bio-tech, Shanghai, China); KLF5 [1:500, a gift from Dr Jin-Tang Dong, Emory University, Atlanta, GA, USA (15)]; poly (ADP-ribose) polymerase (PARP, dilution 1:1,000; cat. no. 9532), caspase-8 (dilution, 1:1,000; cat. no. 9746), caspase-3 (dilution, 1:1,000; cat. no. 9662), JNK (dilution, 1:1,000; cat. no. 9258) and phosphorylated-JNK (dilution, 1:1,000; cat. no. 4668; all from Cell Signaling Technology, Inc. Danvers, MA, USA). Following incubation with the primary antibodies, membranes were incubated with secondary antibody [horseradish peroxidase-conjugated affnipure goat anti-Rabbit IgG (cat. no. ZB-2301; dilution, 1:2,000; OriGene Technologies, Beijing, China) and horseradish peroxidase-conjugated affinipure goat anti-Mouse IgG (cat. no. ZB-2305; dilution, 1:2,000; OriGene Technologies)] for $1 \mathrm{~h}$ at room temperature. Immunoreactive signals were detected using a WesternBright Quantum HRP substrate kit (Advansta, Inc., Menlo Park, CA, USA), visualized by a Molecular Imager ChemiDoc XRS system (Bio-Rad Laboratories, Inc., Hercules, CA, USA). Immunoblotting for GAPDH was performed as an internal control.

Reverse transcription-quantitative PCR (RT-qPCR). RT-qPCR was performed using a previously described method (11). Briefly, total RNA from $2 \times 10^{5}$ LNCaP cells was isolated using TRIzol reagent (Thermo Fisher Scientific, Inc.) and reverse transcribed into cDNA using the PrimeScript ${ }^{\mathrm{TM}}$ RT reagent kit (Takara Biotechnology Co., Ltd., Dalian, China). A total of $50 \mathrm{ng}$ of cDNA was analyzed using a CFX96 real-time PCR system (Bio-Rad Laboratories, Inc.) with SYBR-Green PCR Master mix (Takara Biotechnology Co., Ltd.) to determine the transcriptional expression of specific genes. The thermocycling conditions were as follows: $95^{\circ} \mathrm{C}$ for $30 \mathrm{sec}$, followed by 40 cycles at $95^{\circ} \mathrm{C}$ for $5 \mathrm{sec}$, and $60^{\circ} \mathrm{C}$ for $30 \mathrm{sec}$. GAPDH was used for normalization. Relative gene expression was calculated by the $2^{-\Delta \Delta \mathrm{Cq}}$ method (21). The primer sequences used were as follows: KLF5 forward, 5'-CAGAGGACCTGGTCCAGACAAG-3' and reverse, 5'-GAGGCCAGTTCTCAGGTG 
A
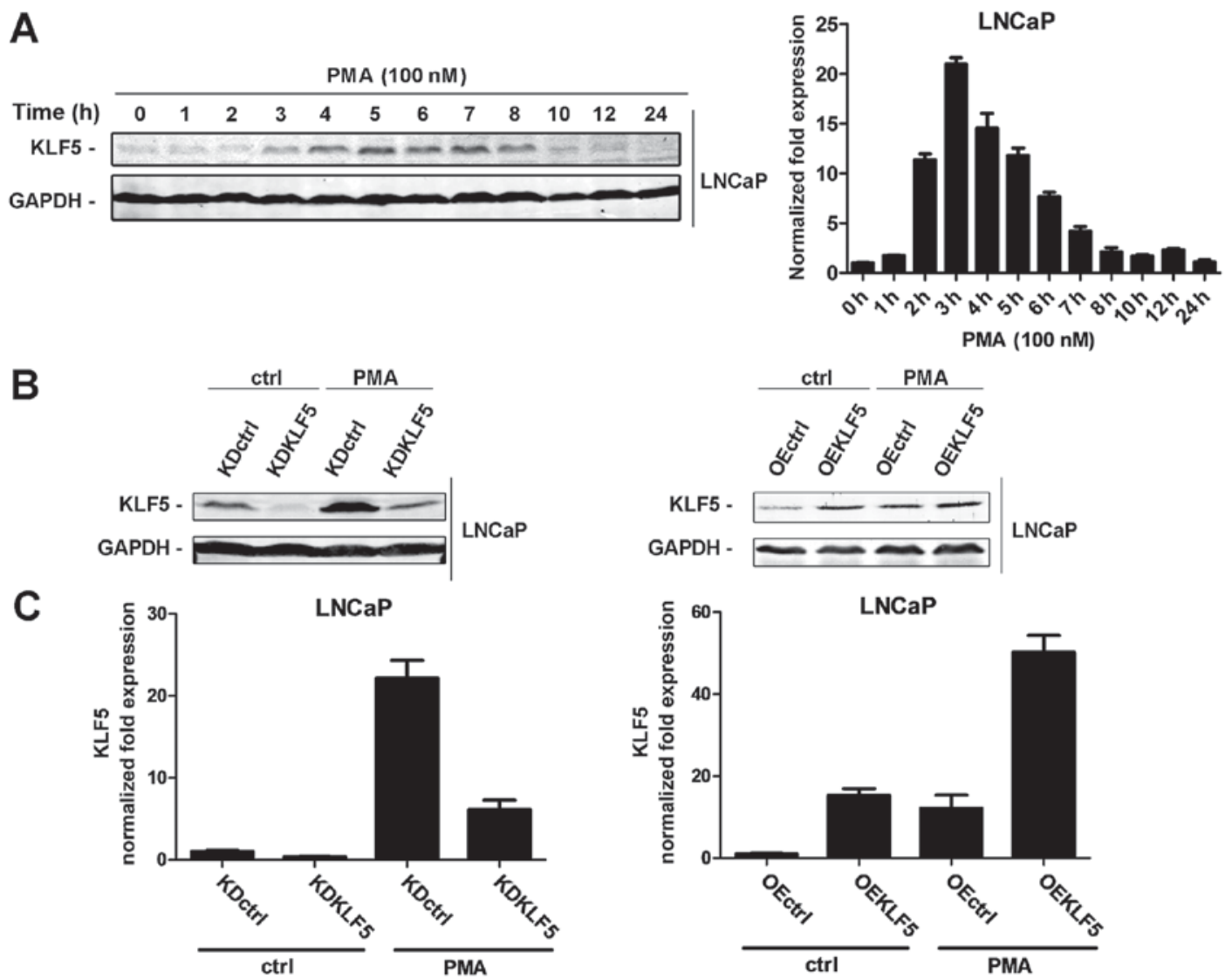

Figure 1. Effect of PMA treatment on the activation of KLF5 in LNCaP cells. (A) LNCaP cells were treated with 100 nM PMA for various amounts of time (0-24 h). KLF5 expression levels were detected at the protein (left) and mRNA (right) levels through western blot analysis and RT-qPCR, respectively. (B) The following LNCaP cell clones were established: Left, KDctrl and KDKLF5; Right, OEctrl and OEKLF5. KLF5 protein expression was determined in the presence or absence of treatment with $100 \mathrm{nM}$ PMA for $4 \mathrm{~h}$ using western blot analysis. (C) Expression of KLF5 mRNA normalized to GAPDH in the following LNCaP clones as detected using RT-qPCR array: Left, KDctrl and KDKLF5; Right, OEctrl and OEKLF5. Similar results were observed in two additional experiments. Data are presented as mean \pm standard deviation following three replicates. RT-qPCR, reverse transcription-quantitative polymerase chain reaction; KDctrl, KLF5 knockdown control; KDKLF5, KLF5 knockdown; OEctrl, overexpression control; OEKLF5, KLF5 overexpression; PMA, phorbol 12-myristate 13-acetate; KLF5, Krüppel-like transcription factor 5; ctrl, control.

AGTG-3'; TNF $\alpha$ forward, 5'-AGCCCATGTTGTAGCAAA CC-3' and reverse, 5'-GGAAGACCCCTCCCAGATAG-3'; GAPDH forward, 5'-ATGGGGAAGGTGAAGGTCGG-3' and reverse, 5'-GACGGTGCCATGGAATT-TGC-3'.

Apoptosis assays. Cells were trypsinized and collected by centrifugation $\left(1,000 \mathrm{x}\right.$ g for $5 \mathrm{~min}$ at $\left.4{ }^{\circ} \mathrm{C}\right)$, and then washed with cold PBS twice. Next cells were stained with Annexin V following the protocol of Annexin-V-FLUOS staining kit (Roche Diagnostics GmbH, Mannheim, Germany). The stained cells were analyzed by fluorescence activated cell sorting using a FACSCalibur ${ }^{\mathrm{TM}}$ flow cytometer (BD Biosciences, Franklin Lakes, NJ, USA) and BD CellQuest Pro software (version 6.0; BD Biosciences).

Collection of conditional medium (CM). Cells were treated with PMA (100 $\mathrm{nM}$ ) or vehicle (DMSO) for $1 \mathrm{~h}$ at room temperature, and then washed twice with RPMI-1640 medium to remove the PMA or DMSO. Following incubation for $24 \mathrm{~h}$, the cell debris was removed from the CM using a $0.22-\mu \mathrm{m}$ Miliex filter (Merck KGaA).

Statistical analysis. GraphPad Prism software (version 5.0; GraphPad, Inc., La Jolla, CA, USA) was used for analyzing differences between two groups using a one-tailed Student's t-test. Data are presented as the mean \pm standard deviation. $\mathrm{P}<0.05$ was considered to indicate a statistically significant difference.

\section{Results}

PMA upregulates KLF5 expression in LNCaP prostate cancer cells. LNCaP cells were treated with $100 \mathrm{nM}$ PMA for various periods of time (0-24 h) and the mRNA and protein expression levels of KLF5 were detected through RT-qPCR and western blot analyses, respectively. As presented in Fig. 1A, KLF5 protein and mRNA expression levels gradually increased throughout the first $0-8 \mathrm{~h}$ of PMA treatment, suggesting that PMA activates KLF5 expression in LNCaP cells. The increase in KLF5 was transient, possibly as the induction of KLF5 is dependent on the phosphorylation of PKC- $\delta$, whose increase is transient.

Establishing the KLF5 knockdown and KLF5 overexpressing LNCAP cell lines. To investigate whether KLF5 is involved in PMA-induced apoptosis of LNCaP cells, stable KLF5 knockdown, KLF5 overexpression cell lines and the corresponding controls were established using lentiviral techniques. 
A

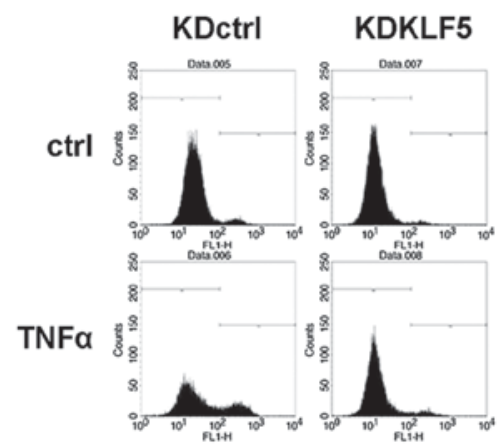

B

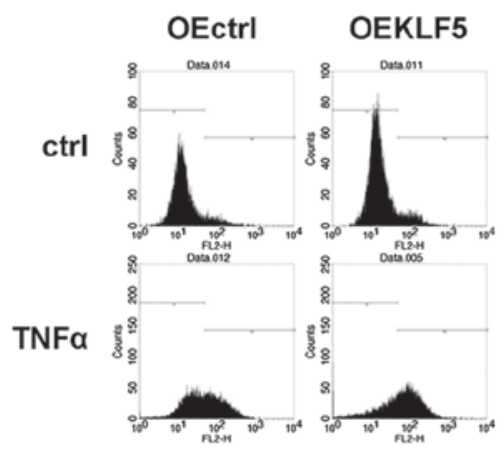

C

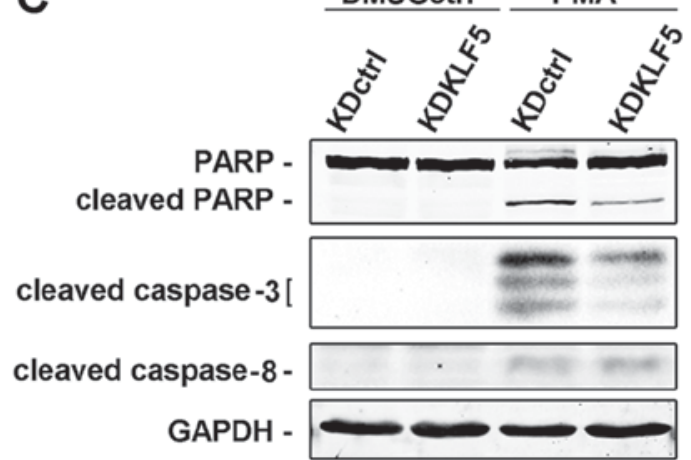

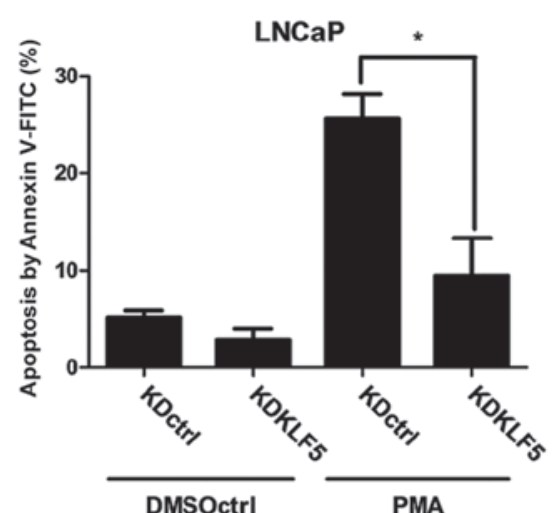

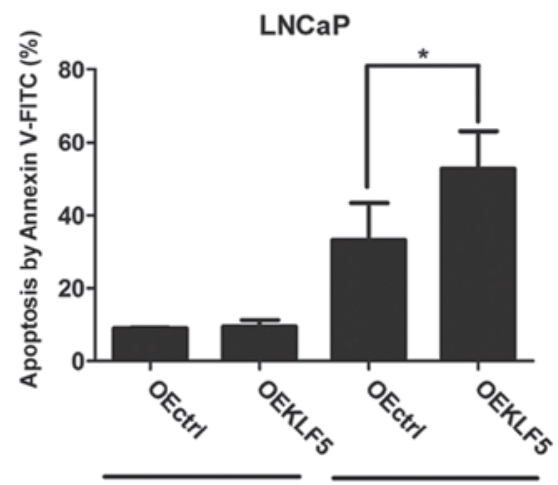

DMSOctrl

D

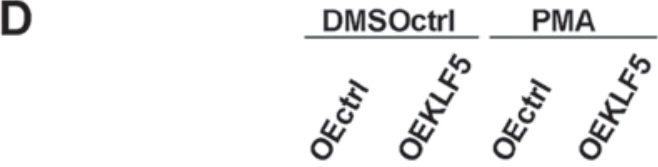
cleaved PARP

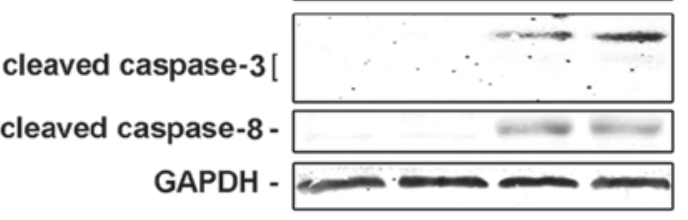

Figure 2. Effect of KLF5 on apoptosis in LNCaP cells induced by PMA. The following LNCaP clones were treated with $100 \mathrm{nM}$ PMA or vehicle control (DMSO) for 24 h: (A) KDctrl and KDKLF5; (B) OEctrl, and OEKLF5. Then the apoptotic cells were detected using flow cytometry analysis. (C) KDctrl and KDKLF5, and (D) OEctrl and OEKLF5 cells were treated with $100 \mathrm{nM}$ PMA or vehicle control for $24 \mathrm{~h}$. Then the differences in specific apoptotic-associated protein expression levels were determined using western blot analysis. Data are presented as mean \pm standard deviation following three replicates. "P $<0.05$. KDctrl, KLF5 knockdown control; KDKLF5, KLF5 knockdown; OEctrl, overexpression control; OEKLF5, KLF5 overexpression; DMSOctrl, DMSO control; PMA, phorbol 12-myristate 13-acetate; KLF5, Krüppel-like transcription factor 5; PARP, poly(ADP-ribose) polymerase.

The expression of KLF5 was verified using western blot and RT-qPCR analyses. The following clones were designed (Fig. 1B and C): KLF5 knockdown control (LNCaP/KDctrl), KLF5 knockdown (LNCaP/KDKLF5), overexpression control (LNCaP/OEctrl) and KLF5 overexpression (LNCaP/OEKLF5).

KLF5 is required for PMA-induced apoptosis in LNCaP cells. Different LNCaP cell clones were treated with $100 \mathrm{nM}$ of PMA for $24 \mathrm{~h}$. Subsequently, cells were harvested and cell apoptosis was determined using flow cytometry. Following stimulation with PMA, the percentage of apoptotic cells was significantly decreased in LNCaP/KDKLF5 compared with LNCaP/KDctrl cells (Fig. 2A), while the cell apoptosis rate was significantly increased in the LNCaP/OEKLF5 group compared with that of the LNCaP/OEctrl group (Fig. 2B).
These results suggest that KLF5 modulates the apoptotic response of LNCaP cells to PMA. Furthermore, the expression levels of proteins involved in the apoptotic cascade following PMA stimulation were measured using western blot analysis. Cleaved PARP and cleaved caspase-3 protein expression levels were decreased following KLF5 knockdown (Fig. 2C), and increased following KLF5 overexpression (Fig. 2D) compared with the corresponding control groups. However, no differences in the expression of cleaved caspase- 8 were observed in the KLF5 knockdown and overexpression groups, indicating that KLF5 knockdown has no significant effect upstream of the TNF $\alpha$ extrinsic apoptosis signaling pathway.

KLF5 is required for CM-PMA-induced apoptosis in LNCaP cells. PMA-induced apoptosis is triggered by the secretion of 
A

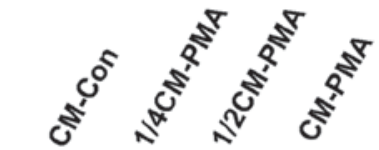

KLF5 -

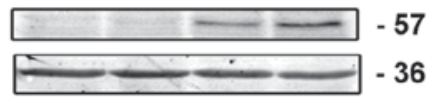

C

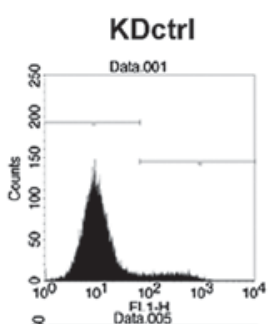

ctrl

PMA
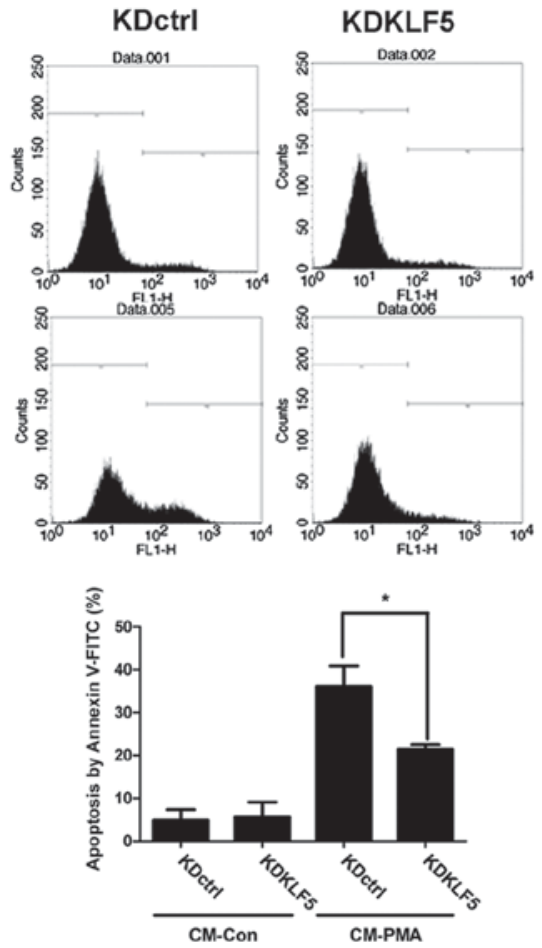

E
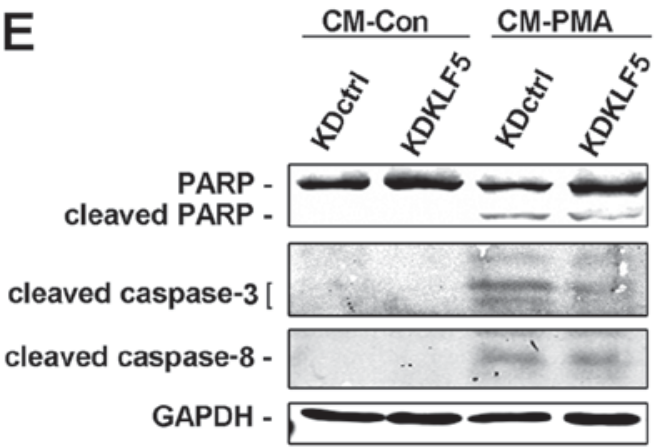

B

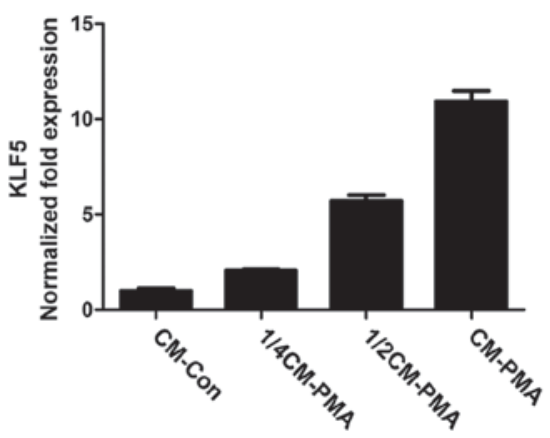

D
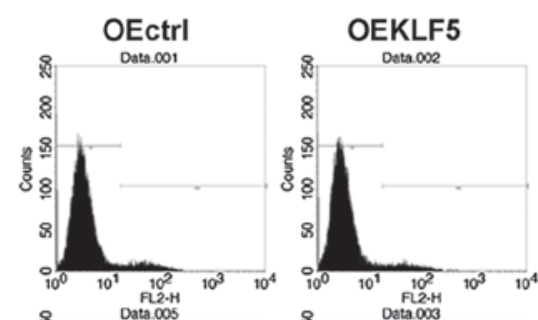

PMA
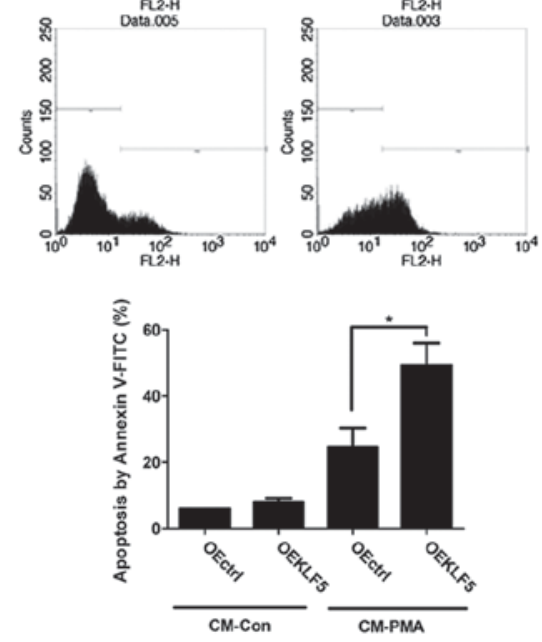

F

LNCaP

cleaved caspase-3 [

cleaved caspase-8 -

GAPDH -

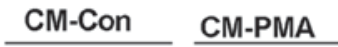

ARP -
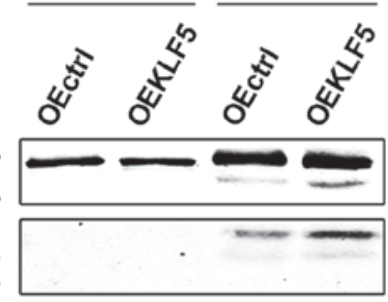

LNCaP

Figure 3. KLF5 is required for CM to induce apoptosis in LNCaP cells. CM from PMA-treated cells enhanced expression of KLF5 (A) protein and (B) mRNA in a dose-dependent manner, as detected using western blot and reverse transcription-quantitative polymerase chain reaction analyses, respectively. (C) Knockdown of KLF5 significantly decreased, but (D) overexpression of KLF5 significantly increased LNCaP cell apoptosis induced by CM-PMA, as detected using flow cytometry assays. Expression of apoptotic proteins in (E) KLF5 knockdown and (F) KLF5-expressing cells treated with CM, as detected through western blot analysis. Similar results were observed in two additional experiments. Data are presented as mean \pm standard deviation following three replicates. "P<0.05. KLF5, Krüppel-like transcription factor 5; CM, control medium; KDctrl, KLF5 knockdown control; KDKLF5, KLF5 knockdown; OEctrl, overexpression control; OEKLF5, KLF5 overexpression; PMA, phorbol 12-myristate 13-acetate; PARP, poly(ADP-ribose) polymerase; CM-Con, control medium from control cells; CM-PMA, control medium from PMA cells.

death factors. Therefore, $\mathrm{LNCaP}$ cells were treated with PMA $(100 \mathrm{nM})$ or vehicle (DMSO) for $24 \mathrm{~h}$, and the CM-PMA and $\mathrm{CM}$-control (Con) was collected. CM-Con and different doses of CM-PMA (dose ratios of CM-Con/CM-PMA were 4:0, 3:1, 2:2, 0:4) were added to a new culture of LNCaP cells. KLF5 protein (Fig. 3A) and mRNA (Fig. 3B) expression levels were increased in a dose-dependent manner following PMA treatment, indicating that KLF5 responds to death factors secreted by PMA-stimulated cells. Furthermore, different LNCaP cell clones were treated with CM-Con and CM-PMA for $24 \mathrm{~h}$ and the apoptotic response were determined using flow cytometry and western blot analysis. The percentage of apoptotic cells 
A

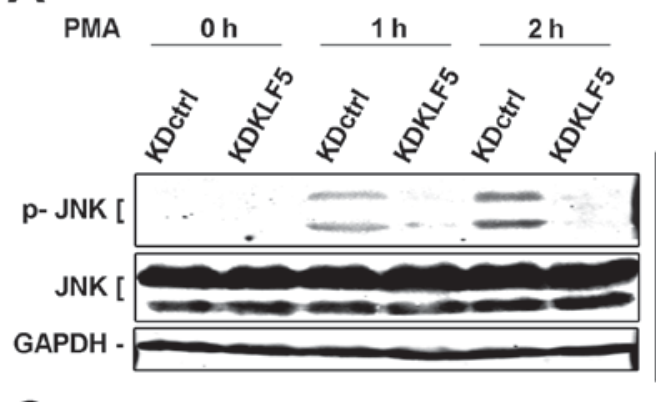

C

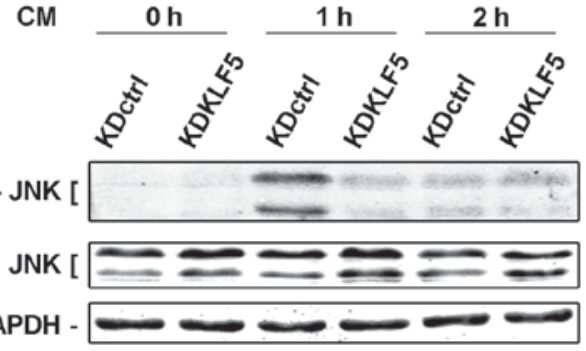

B

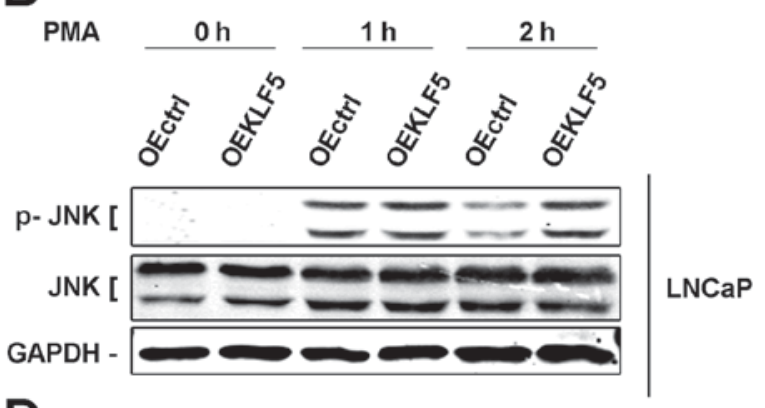

D

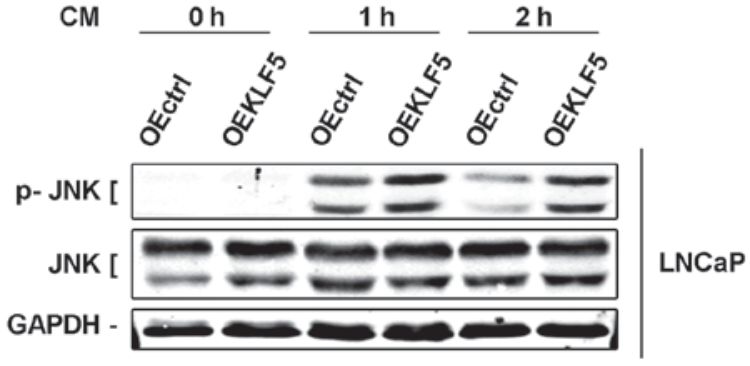

Figure 4. KLF5 modulates apoptosis induced by PMA and CM through regulating the activity of JNK signaling pathway in LNCaP cells. (A) Knockdown of KLF5 decreased, but (B) overexpression of KLF5 increased phosphorylation of JNKs induced by PMA in LNCaP cells. (C) Knockdown of KLF5 decreased, but (D) overexpression of KLF5 increased phosphorylation of JNKs induced by CM in LNCaP cells. Similar results were observed in two additional experiments. Data are presented as mean \pm standard deviation following three replicates. KDctrl, KLF5 knockdown control; KDKLF5, KLF5 knockdown; OEctrl, overexpression control; OEKLF5, KLF5 overexpression; PMA, phorbol 12-myristate 13-acetate; KLF5, Krüppel-like transcription factor 5; CM, control medium; p, phosphorylated; JNK, c-Jun N-terminal kinase.

was significantly decreased in LNCaP/KDKLF5 compared with LNCaP/KDctrl cells (Fig. 3C), while the cell apoptosis was significantly increased in LNCaP/OEKLF5 (Fig. 3D) compared with LNCaP/OEctrl cells. The cleaved PARP and cleaved caspase-3 protein expression levels were decreased in the KLF5 knockdown group (Fig. 3E), and increased following KLF5 overexpression (Fig. 3F) compared with the corresponding control groups. These results suggest that KLF5 is an important molecule for regulating the apoptotic response to death factors secreted by PMA stimulating cells. However, no differences in the expression of cleaved caspase- 8 were observed in the KLF5 knockdown and overexpression groups, indicating that KLF5 is downstream of death factor activation.

KLF5 modulates apoptosis induced by PMA and CM through regulation of the JNK signaling pathway in LNCaP cells. KLF5 has been determined as an important mediator of death factor-induced apoptosis under stimulation of PMA. PMA activates various signaling cascades, in which the JNK signaling pathway is one of the most important apoptotic pathways $(7,22)$. Furthermore, a previous study demonstrated that JNKs are activated by KLF5 (13). Thus, in the present study, the importance of KLF5 to the JNK signaling pathway was investigated. Phosphorylation of JNKs was detected using western blot analysis in different LNCaP cell clones. Following treatment with PMA (0-2 h), the levels of phosphorylation of the JNKs were decreased in LNCaP/KDKLF5 cells (Fig. 4A) and increased in LNCaP/OEKLF5 cells (Fig. 4B) compared with the control groups. Similar results were obtained for LNCaP/KDKLF5 (Fig. 4C) and LNCaP/OEKLF5 (Fig. 4D) cells treated with CM-PMA. These results suggest that
KLF5 modulates the apoptotic response to PMA and CM via regulation of the JNK signaling pathway.

Autocrine factor TNF $\alpha$ mediates the apoptosis induced by $P M A$, independent of KLF5 expression. It has been reported that TNF $\alpha$ is the one of most important autocrine death factors stimulated by PMA in LNCaP cells (7). The stimulation of death receptors activates the extrinsic apoptotic pathway. To confirm the role of TNF $\alpha$, an antibody directed against $\mathrm{TNF} \alpha$ was used to inhibit TNF $\alpha$ expression following PMA or CM-PMA treatment. TNF $\alpha$ inhibition results in decreased KLF5 mRNA expression (Fig. 5A), suggesting that KLF5 is activated by TNF $\alpha$. Furthermore, inhibition of TNF $\alpha$ decreased the PMA- and CM-induced apoptosis in LNCaP cells (Fig. 5B), indicating that autocrine factor TNF $\alpha$ mediates PMA-induced apoptosis, which is consistent with a previous study (7). Furthermore, the involvement of KLF5 in the release of TNF $\alpha$ was investigated. All cell groups were treated with PMA for different times (0-2 h). The release of TNFa protein was determined using western blot analysis (Fig. 5C and D) and the mRNA expression was measured using RT-qPCR analysis (Fig. 5E and F). However, with the presence or absence of PMA, no differences were identified in LNCaP/KDKLF5 or LNCaP/OEKLF5 cells compared with the control groups, suggesting that KLF5 does not regulate the secretion of TNF $\alpha$, and thus does not promote the PMA-induced apoptosis through increasing the expression of $\mathrm{TNF} \alpha$.

\section{Discussion}

Several previous studies have reported that phorbol esters induce an apoptotic response in prostate cancer LNCaP 
A
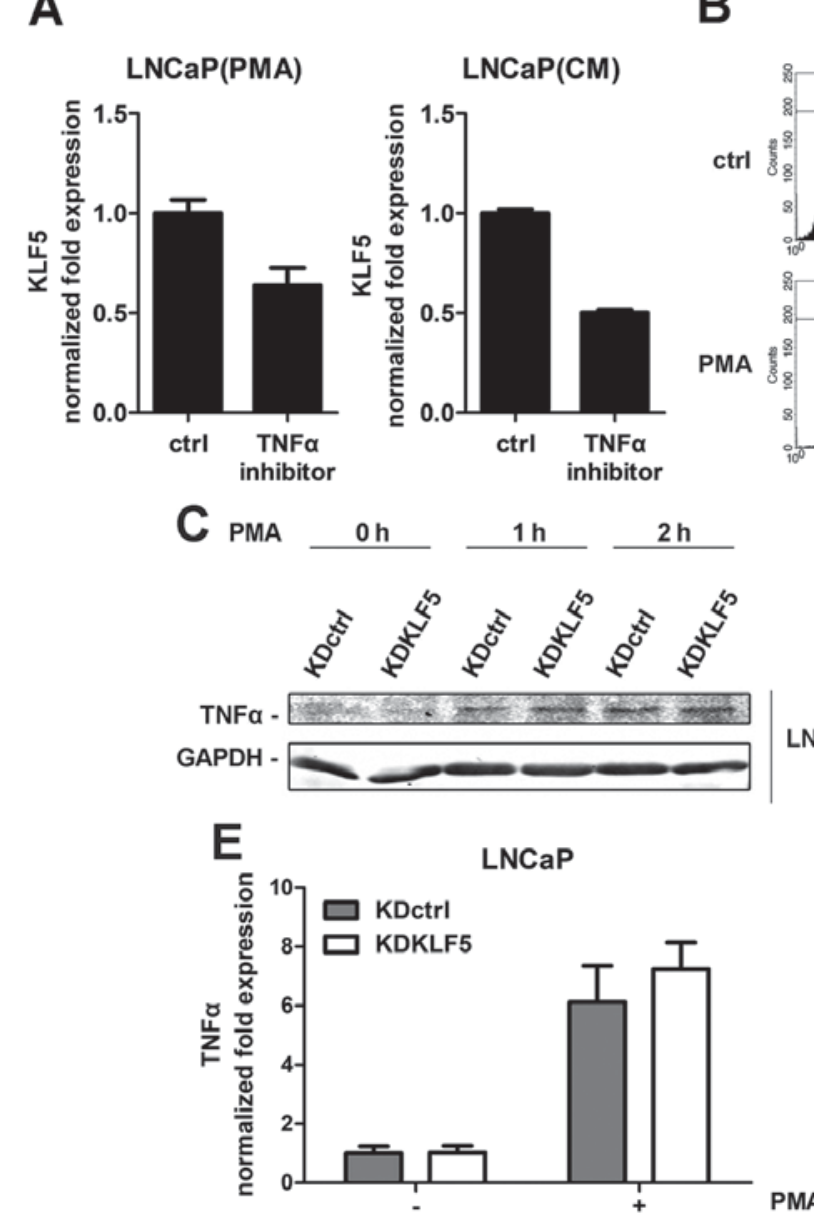

B

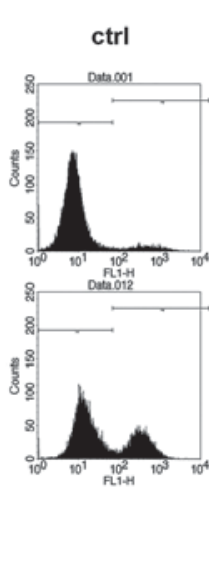

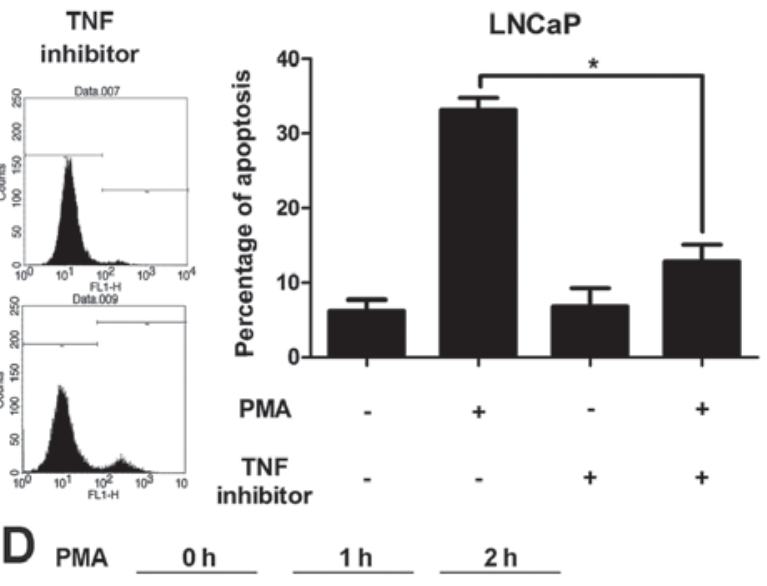

LNCaP

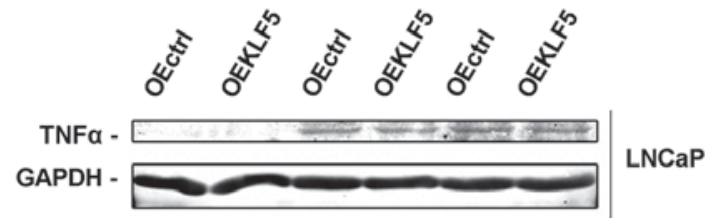

$\mathbf{F}$

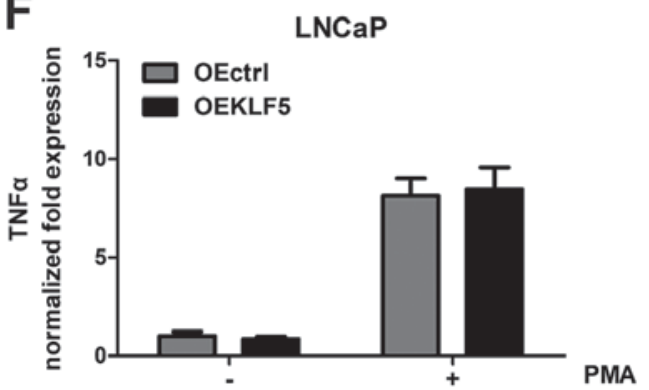

Figure 5. PMA- and CM-induced apoptosis through the autocrine factor TNF $\alpha$. (A) Inhibition of TNF $\alpha$ using an antibody suppressed KLF5 expression induced by PMA and CM, as detected through RT-qPCR arrays. LNCaP cells were treated with PMA or CM for $4 \mathrm{~h}$. (B) TNFa inhibitor suppressed apoptosis induced by PMA and CM, as detected through flow cytometry assays. LNCaP cells were treated with PMA or CM for 24 h. (C) Knockdown of KLF5 and (D) overexpression of KLF5 did not change the expression of TNF $\alpha$ protein induced by PMA treatment in LNCaP cells as detected through western blot analysis. (E) Knockdown of KLF5 and (F) overexpression of KLF5 did not change the expression of TNF $\alpha$ mRNA induced by PMA treatment in LNCaP cells as detected using RT-qPCR arrays and normalized to GAPDH. Cells were treated with $100 \mathrm{nM}$ PMA for $2 \mathrm{~h}$. Similar results were observed in two additional experiments. Data are presented as mean \pm standard deviation following three replicates. ${ }^{*} \mathrm{P}<0.05$. KDctrl, KLF5 knockdown control; KDKLF5, KLF5 knockdown; OEctrl, overexpression control; OEKLF5, KLF5 overexpression; PMA, phorbol 12-myristate 13-acetate; KLF5, Krüppel-like transcription factor 5; CM, control medium; TNF $\alpha$, tumor necrosis factor $\alpha$; RT-qPCR, reverse transcription-quantitative polymerase chain reaction.

cells (4-7). Treatment of LNCaP cells with PMA leads to the autocrine release of death factors, including cytokine $\mathrm{TNF} \alpha(7,8)$. Furthermore, the CM collected from PMA-treated $\mathrm{LNCaP}$ cells has been demonstrated to promote the activation of the extrinsic apoptotic cascade, which activates death effectors, including p38 MAPK, JNK and nuclear factor- $\mathrm{\kappa B}(7,8)$. Inhibition of JNKs has been revealed to decrease the apoptotic effect of PMA $(6,23)$. However, the mechanisms involved remain unclear. In the present study, the results suggest that KLF5 is an essential modulator of PMA-induced apoptosis in LNCaP prostate cancer cells. KLF5 was also identified to be required as an effector of the death factor TNF $\alpha$ and was able to activate the JNK signaling pathway to promote the apoptotic response. Thus, suggesting that KLF5 could be a therapeutic target for prostate cancer. However, the results of the present study revealed that KLF5 exhibited no regulation on the secretion of TNF $\alpha$.

It has been reported that KLF5 has a role in different cell processes, including in cell proliferation, cell cycle progression and apoptosis (9-11). Previous studies have demonstrated that
KLF5 acts as an oncogene in different cancer cells, and is a potential tumor suppressor in prostate cancer cells (24). In the current study, it was revealed that PMA could activate KLF5, and the knockdown or overexpression of KLF5 modulated the apoptotic response to PMA in LNCaP cells. This is similar to the results demonstrated in the CM-treated LNCaP cells, indicating that KLF5 is required for PMA-induced apoptosis.

Death receptors are necessary for the extrinsic apoptotic cascade triggered by death factors, which contain cytoplasmic death domains that enable the receptors to engage the cell apoptotic machinery. Stimulation of these receptors results in the activation of the initiator caspase- 8 , whose cleavage propagates the apoptotic signal (16). However, in the present study, no differences in cleaved caspase-8 expression were identified between KLF5 knockdown or overexpression cells that were treated with PMA or CM and their corresponding control groups. Thus, it may be suggested that stimulation of the death receptor is not influenced by KLF5 or PMA. Furthermore, as TNF $\alpha$ is regarded as the most important autocrine death factor (7), the level of TNF $\alpha$ following PMA stimulation 
was detected. The results revealed that the protein release or mRNA expression of TNF $\alpha$ was not regulated by KLF5; thus, KLF5 may only be an effector of the cytokine TNFa.

The involvement of JNK in various forms of cell death has been reported; however, whether JNK is required for apoptotic cell death induced by TNF $\alpha$ in prostate cancer cells has not been previously elucidated (25). Previous studies have determined that JNK can be activated following phorbol ester treatment in LNCaP cells (7,26). In the current study, KLF5 was detected to regulate phosphorylation of JNKs. Either under stimulation of PMA or treatment with CM, loss of KLF5 was able to significantly decrease the phosphorylation of JNKs, whereas levels were significantly increased in the overexpression group, suggesting that KLF5 is a modulator of JNKs in response to TNF $\alpha$ in LNCaP cells.

In conclusion, the results of the present study suggest that KLF5 is essential for the PMA-induced apoptosis in LNCaP prostate cancer cells. Furthermore, KLF5 is indispensable for the autocrine factor $\mathrm{TNF} \alpha$, which is secreted by cells treated with PMA, to induce cell apoptosis through regulating the activity of JNK signaling pathway. These observations provide novel insights into the complexity of the signaling pathways and the mechanisms regulating cell apoptosis in prostate cancer cells, which could aid in developing novel treatments for patients with prostate cancer.

\section{Acknowledgements}

The present study was supported by the National Natural Science Foundation of China (grant nos. 81672557 and 81372279; received by Peng Guo).

\section{References}

1. Siegel RL, Miller KD and Jemal A: Cancer statistics, 2015. CA Cancer J Clin 65: 5-29, 2015.

2. Mukherji D, Omlin A, Pezaro C, Shamseddine A and de Bono J: Metastatic castration-resistant prostate cancer (CRPC): Preclinical and clinical evidence for the sequential use of nove therapeutics. Cancer Metastasis Rev 33: 555-566, 2014.

3. Barry OP and Kazanietz MG: Protein kinase C isozymes, novel phorbol ester receptors and cancer chemotherapy. Curr Pharm Des 7: 1725-1744, 2001.

4. Garcia-Bermejo ML, Leskow FC, Fujii T, Wang Q, Blumberg PM, Ohba M, Kuroki T, Han KC, Lee J, Marquez VE and Kazanietz MG: Diacylglycerol (DAG)-lactones, a new class of protein kinase $\mathrm{C}$ (PKC) agonists, induce apoptosis in $\mathrm{LNCaP}$ prostate cancer cells by selective activation of PKCalpha. J Biol Chem 277: $645-655,2002$.

5. Tanaka Y, Gavrielides MV, Mitsuuchi Y, Fujii T and Kazanietz MG: Protein kinase $\mathrm{C}$ promotes apoptosis in $\mathrm{LNCaP}$ prostate cancer cells through activation of p38 MAPK and inhibition of the Akt survival pathway. J Biol Chem 278: 33753-33762, 2003

6. Xiao L, Eto M and Kazanietz MG: ROCK mediates phorbol ester-induced apoptosis in prostate cancer cells via p21Cip1 up-regulation and JNK. J Biol Chem 284: 29365-29375, 2009.

7. Gonzalez-Guerrico AM and Kazanietz MG: Phorbol ester-induced apoptosis in prostate cancer cells via autocrine activation of the extrinsic apoptotic cascade: A key role for protein kinase $\mathrm{C}$ delta. J Biol Chem 280: 38982-38991, 2005.
8. Xiao L, Gonzalez-Guerrico A and Kazanietz MG: PKC-mediated secretion of death factors in LNCaP prostate cancer cells is regulated by androgens. Mol Carcinog 48: 187-195, 2009.

9. Guo P, Dong XY, Zhang X, Zhao KW, Sun X, Li Q and Dong JT: Pro-proliferative factor KLF5 becomes anti-proliferative in epithelial homeostasis upon signaling-mediated modification. J Biol Chem 284: 6071-6078, 2009.

10. Gao Y, Wu K, Chen Y, Zhou J, Du C, Shi Q, Xu S, Jia J, Tang X, $\mathrm{Li} \mathrm{F}$, et al: Beyond proliferation: KLF5 promotes angiogenesis of bladder cancer through directly regulating VEGFA transcription. Oncotarget 6: 43791-43805, 2015.

11. Gao Y, Shi Q, Xu S, Du C, Liang L, Wu K, Wang K, Wang X, Chang LS, He D and Guo P: Curcumin promotes KLF5 proteasome degradation through downregulating YAP/TAZ in bladder cancer cells. Int J Mol Sci 15: 15173-15187, 2014

12. Tong D, Czerwenka K, Heinze G, Ryffel M, Schuster E, Witt A, Leodolter S and Zeillinger R: Expression of KLF5 is a prognostic factor for disease-free survival and overall survival in patients with breast cancer. Clin Cancer Res 12: 2442-2448, 2006.

13. Tarapore RS, Yang Y and Katz JP: Restoring KLF5 in esophageal squamous cell cancer cells activates the JNK pathway leading to apoptosis and reduced cell survival. Neoplasia 15: 472-480, 2013.

14. Chen C, Bhalala HV, Vessella RL and Dong JT: KLF5 is frequently deleted and down-regulated but rarely mutated in prostate cancer. Prostate 55: 81-88, 2003.

15. Chen C, Sun X, Ran Q, Wilkinson KD, Murphy TJ, Simons JW and Dong JT: Ubiquitin-proteasome degradation of KLF5 transcription factor in cancer and untransformed epithelial cells. Oncogene 24: 3319-3327, 2005.

16. Aggarwal BB: Signalling pathways of the TNF superfamily: A double-edged sword. Nat Rev Immunol 3: 745-756, 2003.

17. Chopra DP, Menard RE, Januszewski J and Mattingly RR: TNF-alpha-mediated apoptosis in normal human prostate epithelial cells and tumor cell lines. Cancer Lett 203: 145-154, 2004.

18. Shi Q, Gao Y, Xu S, Du C, Li F, Tang XS, Jia J, Wang X, Chang L, He D and Guo P: Kruppel-like factor 5 promotes apoptosis triggered by tumor necrosis factor $\alpha$ in LNCaP prostate cancer cells via up-regulation of mitogen-activated protein kinase kinase 7. Urol Oncol 34: 58.e11-e18, 2016.

19. Bafford R, Sui XX, Wang G and Conte M: Angiotensin II and tumor necrosis factor-alpha upregulate survivin and Kruppel-like factor 5 in smooth muscle cells: Potential relevance to vein graft hyperplasia. Surgery 140: 289-296, 2006.

20. Chen C, Bhalala HV, Qiao H and Dong JT: A possible tumor suppressor role of the KLF5 transcription factor in human breast cancer. Oncogene 21: 6567-6572, 2002.

21. Livak KJ and Schmittgen TD: Analysis of relative gene expression data using real-time quantitative PCR and the 2(-Delta Delta C(T)) Method. Methods 25: 402-408, 2001.

22. Lorenzo PI and Saatcioglu F: Inhibition of apoptosis in prostate cancer cells by androgens is mediated through downregulation of c-Jun N-terminal kinase activation. Neoplasia 10: 418-428, 2008.

23. Gavrielides MV, Gonzalez-Guerrico AM, Riobo NA and Kazanietz MG: Androgens regulate protein kinase Cdelta transcription and modulate its apoptotic function in prostate cancer cells. Cancer Res 66: 11792-11801, 2006.

24. Xing C, Ci X, Sun X, Fu X, Zhang Z, Dong EN, Hao ZZ and Dong JT: Klf5 deletion promotes Pten deletion-initiated luminal-type mouse prostate tumors through multiple oncogenic signaling pathways. Neoplasia 16: 883-899, 2014.

25. Engedal N, Korkmaz CG and Saatcioglu F: C-Jun N-terminal kinase is required for phorbol ester- and thapsigargin-induced apoptosis in the androgen responsive prostate cancer cell line LNCaP. Oncogene 21: 1017-1027, 2002.

26. Ikezoe T, Yang Y, Taguchi H and Koeffler HP: JNK interacting protein 1 (JIP-1) protects LNCaP prostate cancer cells from growth arrest and apoptosis mediated by 12-0-tetradecanoylphorbol-13-acetate (TPA). Br J Cancer 90: 2017-2024, 2004. 